\title{
日本福岛核污染对中国和美国近海影响的集合 统计预测
}

\author{
韩桂军*, 李威, 付红丽, 张学峰, 王喜冬, 吴新荣, 张连新 \\ 国家海洋局海洋环境信息保障技术重点实验室，国家海洋信息中心，天津 300171 \\ *E-mail: gjhan@mail.nmdis.gov.cn \\ 收稿日期: 2012-02-29; 接受日期: 2012-07-08; 网络版发表日期: 2013-04-19 \\ 国家自然科学基金项目(批准号: 41030854, 40906015, 40906016, 41106005, 41176003)资助
}

\begin{abstract}
摘要利用 1978 2011 年的表面漂流浮标和由再分析流场模拟的拉格朗日示踪物, 对日本 福岛核污染影响中国和美国近海的可能时间和影响强度进行了集合统计预测研究. 初步研 究结果表明, 在假定核污染物仅在海洋表层输运的前提下, 示踪物会通过两个输运路径到达 中国近海, 即分别是黑潮回流和副热带环流, 所需时间分别为 1.5 和 3.5 年; 而示踪物到达美 国近海的时间是 4 年. 根据核污染物相对数浓度(单位面积示踪物的个数同初始时刻放射源 处单位面积示踪物个数之比)及其半衰期, 本研究给出了核污染物在上述相应时间尺度上对 中国和美国近海的影响强度分布. 例如, 相对于日本福岛源地核污染物的影响强度初始水 平, 1.5 年以后中国南海 ${ }^{137} \mathrm{Cs}$ 的影响强度为 $1 \%, 4$ 年后上升为 $3 \%$; 而由于强大的黑潮延伸体 流系是核污染物的主要输运路径, 受此影响, 4 年后美国近海 ${ }^{137} \mathrm{Cs}$ 的影响强度为 $4 \%$.
\end{abstract}

\section{关键词}

福岛核污染 集合统计预测 表面漂流浮标 海洋再分析 拉格朗日示踪物
2011 年 3 月 11 日, 即日本地震的第二天, 福岛 核电站的核反应堆开始发生爆炸. 核反应堆的爆炸 导致了大量放射性物质向福岛周边海域泄漏. 2011年 3 月 30 日, 日本中央新闻社报道监测到的放射性污 染物超过本地标准水平的 4000 多倍. 这些核污染物 能否通过海洋环流输运到太平洋沿岸国家引起了世 界关注. 本文主要关注并研究福岛核污染物通过表 层海流的输运对中国和美国近海的影响.

\section{1 方法}

在本研究中, 海洋中放射性污染物被认为是由
许多拉格朗日粒子组成的混合体，每一个拉格朗日 粒子代表一个放射性元素微粒. 这些粒子会沿着水 平和垂直方向运动，但是同周边的海水不发生扩散 或混合过程 ${ }^{[1]}$. 由于海洋中垂向运动相对水平运动非 常小, 并且垂向流速是很难被测量和模拟的, 因此我 们这里不考虑放射性污染物的垂向运动, 并同时假 定这些污染物的拉格朗日粒子仅在海洋表层输运. 据此, 我们可以利用海洋表面漂流浮标和由再分析 流场模拟的拉格朗日示踪物来估计福岛核污染粒子 的拉格朗日输运轨迹和时间尺度.

\section{1 观测数据}

根据 2011 年 3 月 12 15 日期间有关福岛核污染

中文引用格式: 韩桂军, 李威, 付红丽, 等. 日本福岛核污染对中国和美国近海影响的集合统计预测. 中国科学: 地球科学, 2013,43: 831-835

英文引用格式: Han G J, Li W, Fu H L, et al. An ensemble estimation of impact times and strength of Fukushima nuclear pollution to the east coast of China and the west coast of America. Science China: Earth Sciences, 2013, doi: 10.1007/s11430-012-4520-2 
物的相关报道, 我们设定一个以福岛为中心、 $300 \mathrm{~km}$ 为半径的半圆为核污染物的源区 (图 1 中的粉红区域). 这个设定的核污染源区域的空间尺度同黑潮和亲潮 在该区域汇合所引起的混合效应的尺度是一致的 ${ }^{[2]}$. 我们首先统计了 1978 2011 年期间在任意月份途经 核污染源区域的所有海洋表面漂流浮标, 发现共有 176 个浮标经过该区域. 然后利用这些浮标估计核污 染粒子的输运路径和时间尺度. 因为途经上述所定 义的核污染源区域的表面漂流浮标不仅仅是在三月 份, 其他月份的也有, 而福岛核污染物泄漏是发生在 三月份, 所以利用这些浮标仅仅是在年平均流意义 上对福岛核污染物的漂移轨迹和时间尺度的一种近 似估计. 另外, 这些浮标的寿命是完全不同的, 有的 达核污染源区域之后, 不到几个月就停止了工作, 导 致无法确定它们的最终漂移方向. 因此, 实际用来估 计核污染粒子漂移轨迹的表面漂流浮标的个数少于 176. 由于样本数 (表面漂流浮标的个数) 有限, 通过 表面漂流浮标获得的统计结果仅仅给出一个很好的 参考, 或仅表示一种可能性, 缺少统计意义上的可信 度.

\section{2 海洋再分析数据}

国家海洋局海洋环境信息保障技术重点实验室 (Key Laboratory of Marine Environmental Information Technology, MEIT)的 CORA 计划(China Ocean Reanalysis, 中国海洋再分析)建立了全球和中国近海

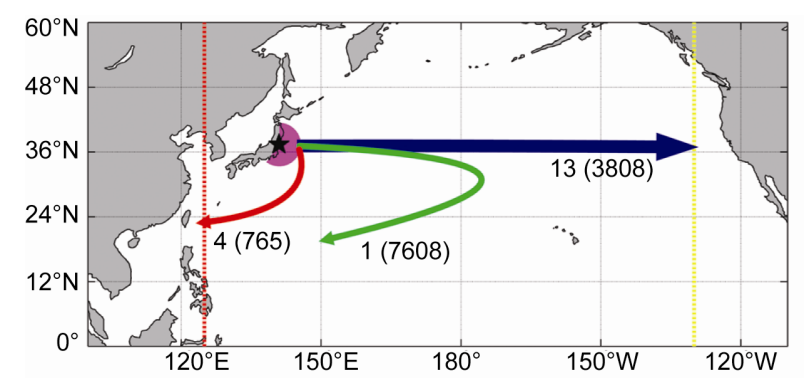

\section{图 1 起源于福岛核污染源区的表面漂流浮标和核污染示} 踪物漂移轨迹示意图

漂移轨迹可分为三种路径: 其一是随着黑潮及黑潮延伸体向东漂移 越过 $130^{\circ} \mathrm{W}$ (黄虚线)到达美国近海(蓝色箭头); 其二是先向东漂移, 然后再转向西漂移, 最终越过 $125^{\circ} \mathrm{E}$ (红虚线) 进入中国近海(绿色箭 头); 其三是直接向西南方向漂移越过 $125^{\circ} \mathrm{E}$ 进入中国近海(红色箭 头). 括号外(内)数字分别表示浮标(示踪物)到达中国近海和美国近 海的个数. 粉红色区域为本研究所设定的以福岛为中心(黑色三角 形)、半径为 $300 \mathrm{~km}$ 的半圆形核污染源区域
及邻近海域海洋再分析系统 ${ }^{[3]}$ (http://www.cora.net. $\mathrm{cn})$. 其中全球海洋模式的区域范围为 $\left(75.25^{\circ} \mathrm{S} \sim\right.$ $\left.84.75^{\circ} \mathrm{N}, 0^{\circ} \sim 360^{\circ} \mathrm{E}\right)$. 全球海洋再分析数据集(CORA) 包含海面高、三维温度、盐度和海流要素, 时间为从 1985 年 1 月 2009 年 4 月共约 25 年, 水平网格分辨 率为 $0.5^{\circ} \times 0.5^{\circ}$, 在赤道附近经向网格加密到 $0.25^{\circ}$, 垂向为 35 层.

检验结果表明, 由于使用了改进的麻省理工学 院海洋环流模式 (the Massachusetts Institute of Technology General Circulation, MITgcm $)^{[4]}$ 和多尺度 三维变分数据同化方法 ${ }^{[57]}$, 上述全球海洋再分析结 果给出了较好的海洋状态场估计. 其中气象驱动场 采用了来自美国国家航天局物理海洋数据分发中心 (PO.DAAC)的 CCMP(a new Cross-Calibrated, MultiPlatform) 风场数据集和美国国家环境预报中心 (National Centers for Environmental Prediction, NCEP) 再分析热通量与淡水通量. 模式的海面温度和盐度 松号到 SODA(Simple Ocean Data Assimilation) ${ }^{[8,9]}$ 的 气候态月平均场, 松弛时间尺度为 100 天. 同化到模 式中的海洋观测数据包括卫星遥感海面温度 (Sea Surface Temperature, SST)、卫星高度计观测的海面高 度异常(Sea Surface Height Anomaly, SSHa), 以及来 自 Argo (Array for real-time geostrophic oceanography) 和 WOD09(World Ocean Database 2009)数据集的温 度和盐度剖面观测。

\section{3 拉格朗日示踪物轨迹}

为了提高样本数量以增强统计意义, 我们使用 了上述 25 年 CORA 表层流场数据来模拟核污染示踪 物, 来进行核污染物输运路径和影响强度的集合统 计预测研究. 我们首先将 5638 个(随机给定)原始示 踪物在 25 年的每年 3 月 11 日随机投放到核污染的源 地(图 1 中的粉色区域), 然后基于 CORA 的表层流再 分析数据, 利用方程(1)即可以获得核污染示踪物的 拉格朗日漂移轨迹.

$$
\left\{\begin{array}{l}
\frac{\mathrm{d} x}{\mathrm{~d} t}=u(x, y, t), \\
\frac{\mathrm{d} y}{\mathrm{~d} t}=v(x, y, t),
\end{array}\right.
$$

其中, $u(x, y, t)$ 和 $v(x, y, t)$ 分别表示格点 $(x, y)$ 在 $t$ 时刻 表层流场的东分量和北分量; $(x, y)$ 表示核污染示踪物 的位置. 
方程(1)的离散形式可以表示为

$$
\left\{\begin{array}{l}
x_{i}\left(t_{n+1}\right)=x_{i}\left(t_{n}\right)+\mathrm{d} t \times u\left(x_{i}\left(t_{n}\right), y_{i}\left(t_{n}\right), t_{n}\right), \\
y_{i}\left(t_{n+1}\right)=y_{i}\left(t_{n}\right)+\mathrm{d} t \times v\left(x_{i}\left(t_{n}\right), y_{i}\left(t_{n}\right), t_{n}\right),
\end{array}\right.
$$

其中, 角标 $i$ 表示第 $i$ 个核污染示踪物; 角标 $n$ 表示时 间积分步; $\mathrm{d} t$ 表示积分步长, 其取值为本研究所使用 的全球海洋再分析的积分时间步长, 即 $7.5 \mathrm{~min} ; t_{n}$ 表 示第 $n$ 个积分步的时间, 即 $n \times \mathrm{d} t+t_{0}, t_{0}$ 为初始时刻.

\section{2 结果分析}

首先, 我们对所有经过核污染源区域的 176 个表 面漂流浮标的轨迹进行逐一分析, 分别统计越过 $125^{\circ} \mathrm{E}$ (图 1 中红色虚线)进入中国近海区域和越过 $130^{\circ} \mathrm{W}$ (图 1 中黄色虚线)进入美国近海区域的浮标个 数以及轨迹的种类. 分析结果表明, 浮标漂移轨迹可 归为三个路径, 分别如图 1 中红色、绿色和蓝色箭头 所示. 途经核污染源区域的漂流浮标中有 $80 \%$ (141 个) 的浮标随黑潮及黑潮延伸体向东漂移, 仅有 20\%(35 个)的浮标向南漂移. 在随黑潮及黑潮延伸体 向东漂移的 141 个浮标中, 其中有 13 个最终能越过 $130^{\circ} \mathrm{W}$ 到达美国近海, 如图 1 中蓝色箭头所示. 图 2 中的蓝色曲线给出这 13 个浮标中的 5 个浮标的漂移 轨迹图. 在向东漂移的 141 个浮标中, 有部分浮标在 漂移过程中停止了工作, 无法确定它们最终是否能 到达美国近海. 在向南漂移的 35 个浮标中, 只有 5 个浮标转向西漂移到达中国近海, 且其中有 1 个浮标 通过吕宋海峡进入中国南海, 如图 2 中红色和绿色曲 线所示. 同样, 在向南漂移的 35 个浮标中, 也有部分

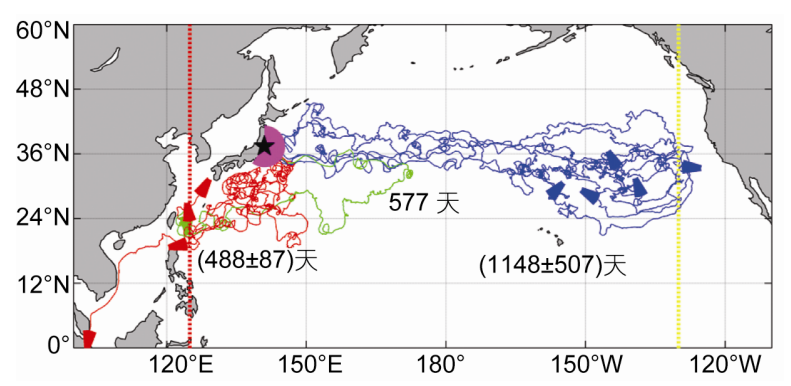

\section{图 2 起源于核污染源区的表面漂流浮标最终漂移到中国 近海的轨迹}

中国近海 5 个, 4 个(红色曲线) 随黑潮回流漂移, 1 个(绿色曲线) 随中 部太平洋的副热带环流漂移; 美国近海 13 个, 这里只显示其中的 5 个(蓝色曲线). 数字分别表示浮标通过每个路径到达目的地的平均 时间和标准差
浮标在漂移过程中停止了工作, 无法确定它们最终 是否能到达中国近海. 在到达中国近海的 5 个浮标中, 有 1 个浮标是随着中部太平洋的副热带环流漂移进 入中国近海的, 其漂移的路径相对比较长(图 2 中绿 色曲线); 其余 4 个浮标是随着黑潮回流(recirculation gyre)漂移进入中国近海(图 2 红色曲线).

我们统计所有模拟的核污染示踪物的漂移轨迹, 发现示踪物漂移进入美国近海(图 3 中蓝色曲线)和中 国近海(图 3 中红色和绿色曲线)的轨迹种类同表面漂 流浮标的漂移轨迹种类是一致的, 但每一类轨迹的 样本数(图 1 括号中数字所示)大大增加, 其中 3808 个 示踪物进入美国近海, 8373 个示踪物进入中国近海. 通过数值模拟极大地增加了集合统计预测的样本数, 特别是随着中部太平洋副热带环流漂移进入中国近 海的轨迹样本数增加到 7608 个 (观测到的这样的表面 漂流浮标轨迹只有 1 个). 此外, 由于核污染源处所有 示踪物是在每年的 3 月份随机投放的，同表面漂流浮 标相比, 利用这些示踪物来估计 3 月份福岛核污染物 的输运路径和时间尺度将更合理.

基于表面漂流浮标和模拟的核污染示踪物的漂 移时间，可以估计核污染物达到中国近海和美国近 海的时间尺度, 结果如图 2 和 3 中成对数字所示(观测 到的随中部太平洋的副热带环流漂移的浮标轨迹除 外). 其中, 成对数字分别表示每一种输运路径的平 均漂移时间和标准偏差.

总的来说，基于再分析表层流场模拟的核污染 示踪物所估计的时间尺度比基于观测的表面漂流浮 标所估计的时间尺度更长一些. 这种差异显然同样 本的多少和再分析数据的质量影响拉格朗日积分精 度有关. 如果只保留三月份途经核污染源并且漂移

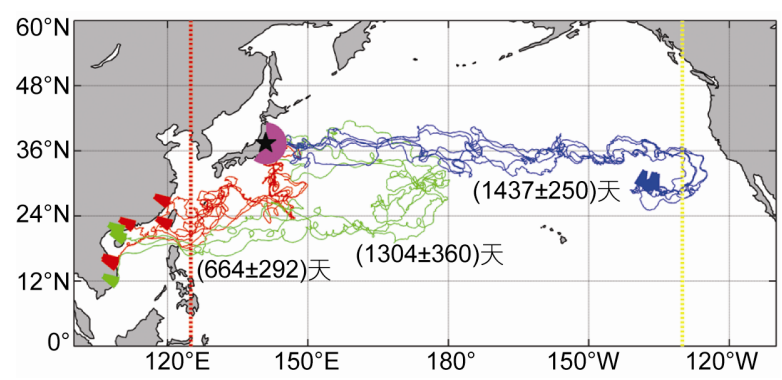

图 3 利用再分析表层流场模拟的核污染示踪物最终到达中 国近岸(红色和绿色)或美国近岸(蓝色)的漂移轨迹的个例图 数字分别表示每个路径中所有示踪物到达目的地的平均时间和标 准差 
寿命足够长的表面漂流浮标, 并且这些浮标样本的 数量足够多的话, 那么通过它们获得的估计结果将 会更可信. 同样, 再分析表层流场的精度越高, 通过 示踪物获得的估计结果也将会更可信. 在本研究中, 基于表面漂流浮标估计的核污染物通过中部太平洋 的副热带环流漂移进入中国近海的漂移时间尺度的 不确定性最大, 由这 1 个表面漂流浮标所估计的时间 尺度为 1.6 年, 而通过 7608 个核污染示踪物所估计的 时间尺度为 3.6 年. 对于其他两种漂移路径, 所估计 的输运时间尺度的不确定性相对要小一些. 例如, 基 于表面漂流浮标所估计的核污染物到达美国近海的时 间尺度为 3.2 年，而基于核污染示踪物所估计的时间 尺度为 3.9 年; 基于仅在西太平洋区域漂移的表面漂 流浮标所估计的核污染物输运到达中国近海的时间尺 度为 1.3 年, 而基于核污染示踪物所估计的时间尺度 为 1.8 年. 这样的结果表明, 利用再分析流场模拟核污 染示踪物以便增加样本数量是一种有效的手段.
基于再分析表层流场模拟核污染示踪物，使得 我们可以利用集合统计预测的方式估计核污染物对 中国和美国近海在不同时间尺度上的影响强度分布. 在本研究中, 海洋中的核污染物被看成是许多拉格 朗日粒子的集合体，它们的浓度可以通过数浓度表 达, 即等于单位面积内核污染示踪物的个数 ${ }^{[1]}$. 在任 意时刻(相对于福岛核污染物发生泄漏的初始时刻), 我们可以计算核污染示踪物的相对数浓度, 其等于 示踪物的数浓度同核污染源处初始时刻示踪物的数 浓度之比. 核污染物在某时刻的影响强度可以用示 踪物的相对数浓度同相应放射性元素在该时刻的衰 减率之积表示.

报道显示，在福岛周边海域内的放射性元素 ${ }^{131} \mathrm{I}$ (半衰期大约为 8 天)、 ${ }^{137} \mathrm{Cs}$ (半衰期大约为 30 年) 和 ${ }^{134} \mathrm{Cs}$ (半衰期大约为 2 年)严重超标. ${ }^{137} \mathrm{Cs}$ 的半衰期 很长, 它将会对人类产生更大的危害. 图 4 分别给出 ${ }^{137} \mathrm{Cs}$ 在福岛核污染泄漏后 1.5 年(图 4(a))、3.5 年(图
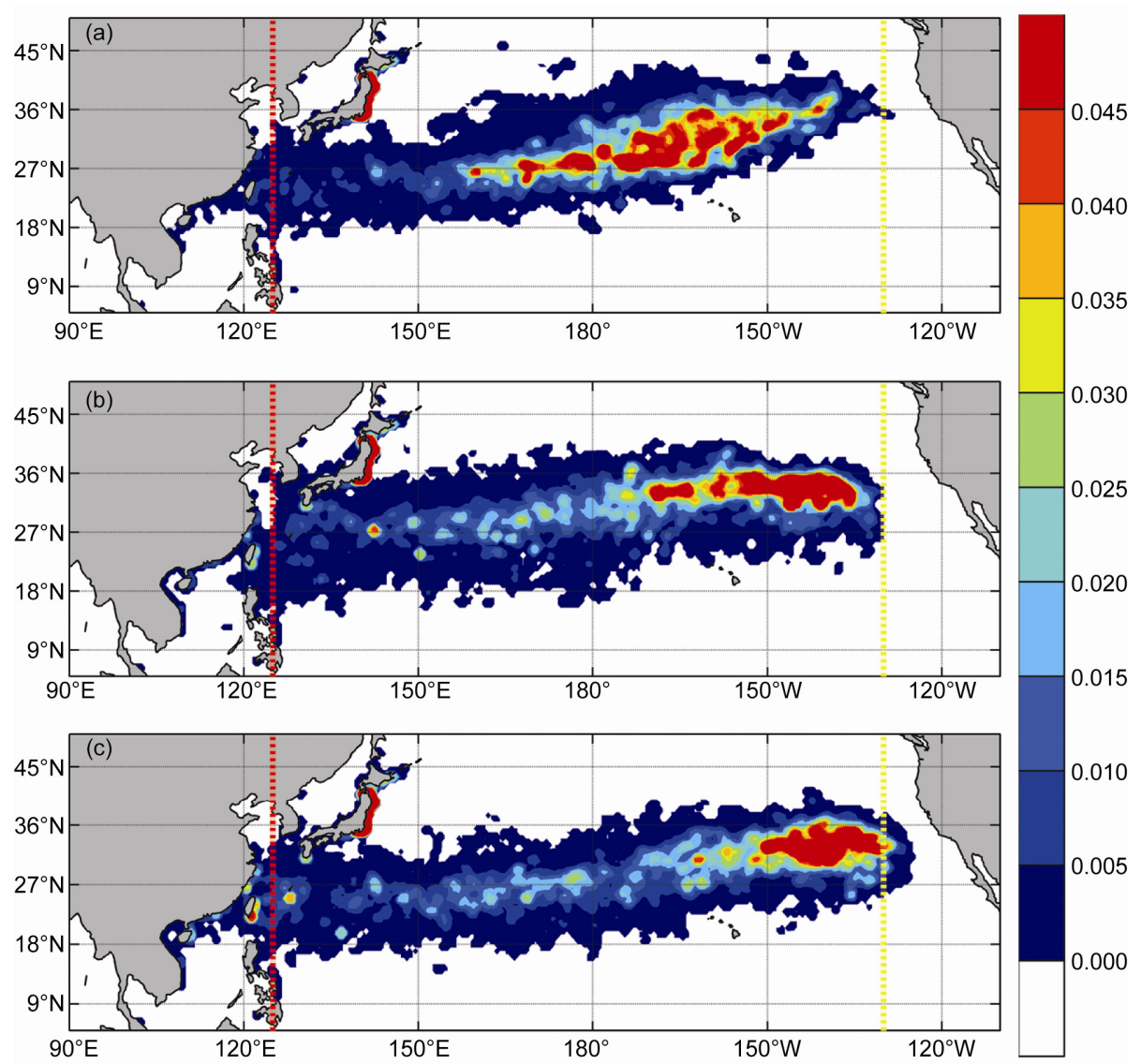

图 $4{ }^{137} \mathrm{Cs}$ 影响强度的空间分布

(a), (b)和(c)分别对应日本福岛核泄漏发生后的 $1.5,3.5$ 和 4 年 
4(b))和 4 年(图 4(c))的影响强度分布. 由图 4 可以看 出, 相对于日本福岛源地核污染物的影响强度初始 水平, 1.5 年以后中国南海 ${ }^{137} \mathrm{Cs}$ 的影响强度为 $1 \%$, 受 局地涡旋的影响, 4 年后上升为 $3 \%$. 需要指出的是, 由于全球再分析结果不能很好地构建近岸附近的流 场, 一些核污染示踪物可能会一直停留在近岸区域, 这将造成该区域内影响强度估计的不确定性. 由于 强大的黑潮延伸体流系是核污染物的主要输运路径, 受此影响, 4 年后美国近海 ${ }^{137} \mathrm{Cs}$ 的影响强度为 $4 \%$.

\section{3 讨论和结论}

根据 2011 年 3 月 12 15 日期间福岛核污染物泄 漏事件的有关新闻报道, 本研究设定以福岛为中心、 $300 \mathrm{~km}$ 为半径的半圆作为核污染源区域，统计 1978 2011 年期间所有途经该核污染源区域的表面 漂流浮标(共计 176 个)轨迹, 用以估计福岛核污染物 通过海流输运到中国和美国近海的时间尺度与影响 强度. 为了增加统计的样本数, 本研究利用约 25 年 的再分析表层流场来模拟核污染示踪物.

统计结果显示, 核污染示踪物需要花费大约 4 年 的时间才能到美国近海; 而漂移到达中国近海的示
踪物轨迹主要有两类: 一类是随黑潮回流, 大约需要 1.5 年的时间; 另一类是随中部太平洋的副热带环流, 大约需要 3.5 年的时间. 在上述不同时间尺度上核污 染物影响强度的空间分布可以用核污染示踪物的相 对数浓度与相应的放射性元素衰减率之积来表示. 结果显示, 相对于日本福岛源地核污染物的影响强 度初始水平, 1.5 年以后中国南海 ${ }^{137} \mathrm{Cs}$ 的影响强度为 $1 \%$, 受局地涡旋的影响, 4 年后上升为 $3 \%$; 而由于强 大的黑潮延伸体流系是核污染物的主要输运路径, 受此影响, 4 年后美国近海 ${ }^{137} \mathrm{Cs}$ 的影响强度为 $4 \%$.

虽然本文初步的研究结果表明，基于表面漂流 浮标获得的统计结果与基于再分析流场模拟的示踪 物获得的统计结果基本上是一致的，但表面漂流浮 标的个数和再分析数据的精度对统计结果的准确性 具有较大影响. 在后续研究中, 通过增加观测数据的 样本数, 以及使用不同的再分析数据和分析不同统 计结果的差异, 可以进一步增强对核污染物运输机 制的理解，提高对输运时间尺度和影响强度的估计 精度. 另外，本文中对观测的浮标和模拟的示踪物的 分析也还需要进一步深入, 尤其是要考虑到海洋三 维流场的影响.

\section{参考文献}

1 刘爱华, 萠琳萍. 放射性核素大气弥散模式研究综述. 气象与环境学报, 2011, 27: 59-65

2 Shimizu Y, Yasuda I, Ito S-I. Distribution and circulation of the coastal Oyaishio intrusion. J Phys Oceanogr, 2001, 31: 1561-1578

3 Han G J, Li W, Zhang X F, et al. A regional ocean reanalysis system for coastal waters of China and adjacent seas. Adv Atmos Sci, 2011, 28: $682-690$

4 Marshall J, Hill C, Perelman L, et al. Hydrostatic, quasi-hydrostatic and nonhydrostatic ocean modeling. J Geophys Res, 1997, 102: 5733-5752

5 Li W, Xie Y, He Z J, et al. Application of the multi-grid data assimilation scheme to the China Seas' temperature forecast. J Atmos Ocean Technol, 2008, 25: 2106-2116

6 Li W, Xie Y, Deng S-M, et al. Application of the multigrid method to the two-dimensional doppler radar radial velocity data assimilation. J Atmos Ocean Technol, 2010, 27: 319-332

7 Xie Y, Koch S, Mcginley J, et al. A space-time multiscale analysis system: A sequential variational analysis approach. Mon Weather Rev, 2011, 139: 1224-1240

8 Carton J A, Chepurin G, Cao X. A simple ocean data assimilation analysis of the global upper ocean 1950-95. Part I: Methodology. J Phys Oceanogr, 2000, 30: 294-309

9 Carton J A, Chepurin G, Cao X. A simple ocean data assimilation analysis of the global upper ocean 1950-95. Part II: Results. J Phys Oceanogr, 2000, 30: 311-326 